\title{
Tunable plasmonics by self-assembled stretchable superlattices on macroscopic scale
}

\author{
Mathias Charconnet ${ }^{1,2}$, Christian Kuttner ${ }^{2}$, Cristiano Matricardi ${ }^{3}$, Agustín Mihi ${ }^{3}$, Luis M. Liz-Marzán ${ }^{2,4}$, Andreas Seifert ${ }^{1,4}$ \\ ${ }^{1}$ CIC nanoGUNE, Donostia-San Sebastián, Spain, ${ }^{2}$ CIC biomaGUNE, Donostia-San Sebastián, Spain \\ ${ }^{3}$ Institut de Ciència de Materials de Barcelona (ICMAB-CSIC), Bellaterra, Spain \\ ${ }^{4}$ IKERBASQUE, Basque Foundation for Science, Bilbao, Spain \\ m.charconnet@nanogune.eu,ckuttner@cicbiomagune.es,cmatricardi@icmab.es,amihi@icmab.es \\ llizmarzan@cicbiomagune.es,a.seifert@nanogune.eu
}

\begin{abstract}
We present a process to create flexible 2D superlattices of nanoparticles, self-assembled into discrete and long-range ordered clusters. The process is readily scalable and grants access to mechano-responsive nanostructures on square centimeter areas. The elastic properties of the support allow for post-assembly tailoring of the lattice periodicity. The corresponding change in collective lattice coupling results in a gradual shift of the extinction maximum upon mechanical strain. Our results give a proof-of-principle for tunable plasmonics by mechano-responsive nanostructures.
\end{abstract}

Keywords - lattice plasmons, mechano-plasmonics, selfassembly, gold nanoparticles, enhanced spectroscopy

\section{INTRODUCTION}

Plasmonic substrates are powerful tools for enhanced spectroscopy, such as Raman spectroscopy or localized surface plasmon resonance (LSPR) sensing. However, high signal intensities demand matching of the plasmon resonance with the excitation wavelength [1]. Periodical arrangements of nanoparticles (NPs), so-called superlattices, feature intensive lattice plasmons because of long-range ordered domains. Such lattice plasmons arise from an interference between the LSPR of the individual domains and an in-plane diffracted wave, the so-called Rayleigh anomaly. As a consequence, the optical response of superlattices shows high sensitivity on the lattice period [2]. Superlattices on flexible supports would grant access to tunable plasmonic. So far, the methods reported focus on the use of bulk gold nanostructures made by electron-beam lithography (EBL) [3]. EBL is inherently a slow and expensive method and not readily compatible with flexible substrates. We present here a fast and scalable process to create superlattices of NPs on a flexible substrate. The optical properties of such substrates upon mechanical strain will be discussed in detail and compared to reference substrates of defined periodicities.

\section{RESUlTS AND DisCUSSION}

\section{A. Fabrication process}

We use a templated self-assembly process to fabricate plasmonic substrates. This process is described in Fig. 1a [4]. A droplet of NP dispersion is cast on a glass slide or PDMS (polydimethylsiloxane) slab. A droplet of a colloidal dispersion of gold nanospheres (water, ethanol, surfactant, NPs@poly(ethylenglycole)) is confined between a nanostructured template, made of PDMS and the target substrate (Fig. 1a). The template is composed of an array of squared wells (Fig. 1b). Capillary forces guide the NPs into the holes of the template, yielding a superlattice of NPs after drying. The size of the domains can be controlled by the size/volume ratio of the template structure and the NP size $[1,4,5]$. Here, we use PDMS both as template mold as well as a soft flexible target substrate. For that purpose, the PDMS

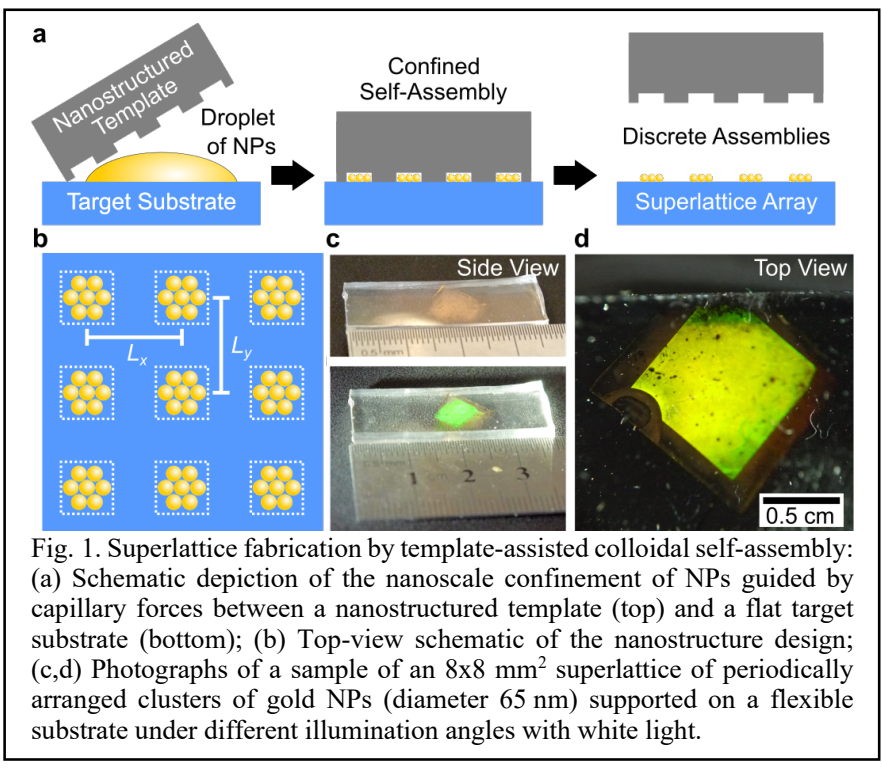

target surface needs to be functionalized with a thin layer of a few nanometers of polyethyleneimine (PEI, branched, $25 \mathrm{~kg} / \mathrm{mol}$ ). PEI inhibits a cohesive contact between template and substrate and promotes the transfer of NPs from the mold to the substrate. [5]. Fig. 1c shows the angle-dependent reflection of a superlattice illuminated with white light. The green color is caused by interaction of the white light with the periodical arrangements of NPs (Fig. 1d). Using EBL for nanostructuring of such an area of around $8 \times 8 \mathrm{~mm}^{2}$ would take several tens of hours.

\section{B. Macroscopic strain-induced lattice deformations}

Upon stretching, a gradual color shift from green (100\%) to red (128\% elongation) can be observed (Fig. 2a). This optical change in reflection can be explained by the change in periodicity of the nanosphere clusters. Fig. $2 b$ depicts the deformation of the lattice induced by macroscopic strain. The effect of lattice variation was investigated by SEM imaging for a superlattice of $65 \mathrm{~nm}$ NPs of $500 \mathrm{~nm}$ period fabricated by the templated self-assembly process described in Part A (Fig. 2c). Elongated to $148 \%$, the distances between the clusters are considerably augmented from 500 to $900 \mathrm{~nm}$ (Fig. 2d), however, after elongation, the clusters by themselves were found to remain close-packed. This robustness is an important point for keeping the plasmonic properties of the sample unperturbed. The robustness of assemblies is controlled by the balance of cohesive and adhesive interactions at the interface. Assemblies of similar NP sizes and surface chemistry were found to experience structural changes upon elongation to $130 \%$ [5]. 


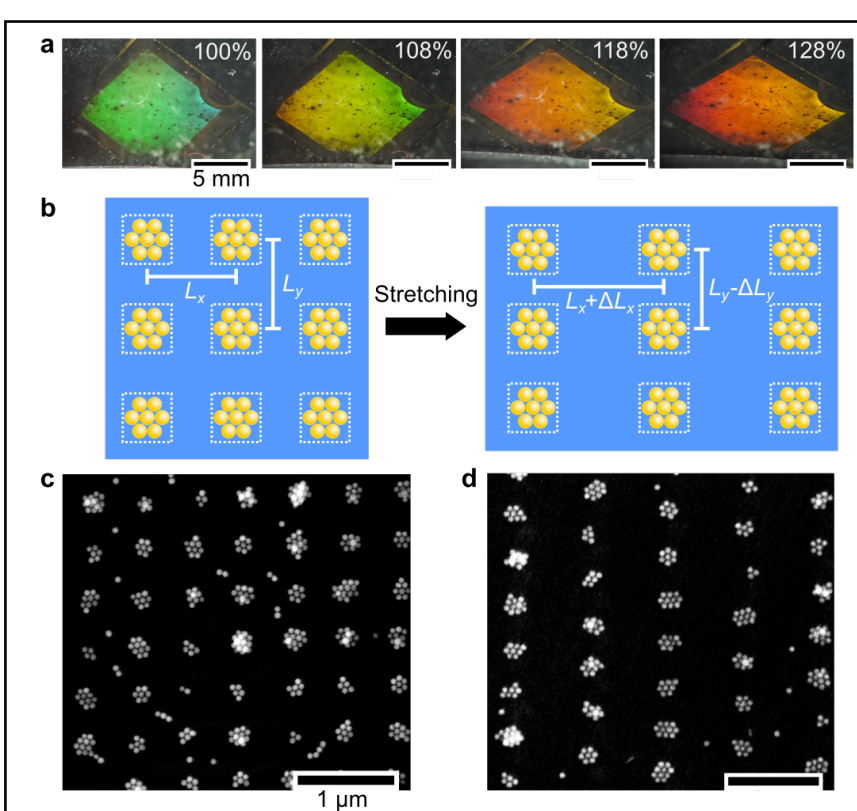

Fig. 2. Macroscopic strain-induced lattice deformation: (a) Gradual color change from green to red upon elongation under white light illumination. (b) Schematic depiction of the lattice deformation and SEM images in (c) unstretched and (d) stretched state.

As a reference experiment, we used nanostructured PDMS molds of 500 and $600 \mathrm{~nm}$ periods to fabricate superlattices of the corresponding periodicities. The extinction of superlattices of 500 and $600 \mathrm{~nm}$ spacings is plotted in Fig. 3a. The main resonances are located at $750 \mathrm{~nm}$ and $900 \mathrm{~nm}$, respectively. This redshift can be attributed to changes in farfield coupling because of the increased lattice spacing $[1,2]$. The main advantage of having a flexible substrate is that the lattice parameter of a substrate could be adjusted simply and reversibly by stretching, thus, allowing tuning of the plasmonic response. Consequently, the lattice plasmon of choice could be tailored without the need to fabricate the corresponding mold geometries.

\section{Tunable plasmonics}

Fig. 3a shows the optical response of a superlattice of $500 \mathrm{~nm}$ in an unstretched state and at $108 \%, 118 \%$, and $128 \%$ of elongation. Initially, the unstretched sample shows a plasmon resonance at around $780 \mathrm{~nm}$, as expected for a sample of $500 \mathrm{~nm}$ period. Upon further elongation at approx. $115 \%$, a redshift of the plasmon resonance starts to become apparent. The resonance continues to shift linearly toward the red (Fig. 3c). Precisely, the extinction maximum may be tuned from $795(100 \%)$ to $971 \mathrm{~nm}$ (at $160 \%$ ). At the same time, the high-energy signature below $700 \mathrm{~nm}$ seems to remain almost completely unperturbed. This spectral range is expected to be dominated by the ensemble-averaged optical properties of the individual clusters. If so, this would give further proof for the high structural robustness of the particle assembly - even upon high strain values.

In a next step, we correlate the lattice plasmon shift obtained by stretching with reference substrates fabricated using molds of corresponding lattice periodicities. Superlattices of $600 \mathrm{~nm}$ period support a lattice plasmon at $900 \mathrm{~nm}$ (Fig. 3a). To yield the same lattice plasmon, the flexible superlattice of initially $500 \mathrm{~nm}$ periodicity need to be stretched to approx. $120 \%$, which is equivalent to a horizontal periodicity of $600 \mathrm{~nm}$. This is in excellent agreement with the uniaxial deformation of a square lattice to a rectangular lattice. The overall optical change upon uniaxial

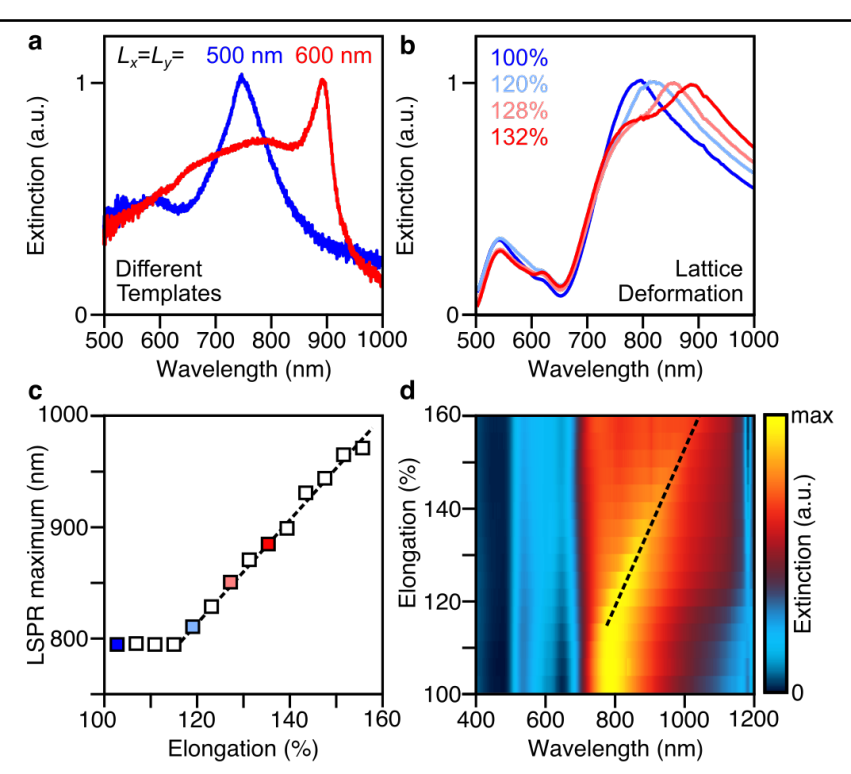

Fig. 3. Tunable plasmonics: (a) Normalized extinction spectra of samples made with molds of 500 and $600 \mathrm{~nm}$. (b) Evolution of the Normalized maximum extinction with elongation. (c) Maximum extinction of $65 \mathrm{~nm}$ nanosphere clusters in a $500 \mathrm{~nm}$ lattice at different elongation. (d) 2D map of the optical response of a $65 \mathrm{~nm}$ nanosphere cluster in a $500 \mathrm{~nm}$ lattice.

deformation was monitored by a series of UV/vis spectra (Fig. 3d). The non-normalized 2D map highlights the mechanoresponsive shift of the lattice plasmon (yellow) for the increasing periodicity.

\section{CONCLUSION}

We have presented a templated self-assembly process that allows formation of plasmonic superlattices on a flexible support. These superlattices show increased robustness upon stretching and maintain the optical properties of each clusters. Thanks to this robustness, the plasmonic response can be finetuned to the excitation wavelength of choice.

\section{ACKNOWLEDGMENT}

This work was supported by the Spanish Ministry of Economy, Industry and Competitiveness under the Maria de Maeztu Units of Excellence Programme - MDM-2016-0618 and by the Basque Government in the Elkartek program 2018/19. C.K. acknowledges funding from the European Union's Horizon 2020 research and innovation programme under the Marie Skłodowska-Curie grant agreement No. 799393 (NANOBIOME).

\section{REFERENCES}

[1] C. Matricardi et al., "Gold Nanoparticle Plasmonic Superlattices as Surface Enhanced Raman Spectroscopy Substrates," ACS Nano, vol. 12, pp. 8531-8539, 2018.

[2] V. Kravets, Q. V. Kabashin, W. L. Barnes, A.N. Grigorenko, "Plasmonic Surface Lattice Resonances: a Review of Properties and Applications.” Chem. Rev., vol. 118, pp. 5912-5951, 2018.

[3] M. L. Tseng et al., "Two-Dimensional Active Tuning of an Aluminum Plasmonic Array for Full-Spectrum Response," Nano Lett., vol. 17, pp. 6034-6039, 2017.

[4] C. Hanske et al., "Solvent-Assisted Self-Assembly of Gold Nanorods into Hierarchically Organized Plasmonic Mesostructures," ACS Appl. Mater. Interfaces, DOI: 10.1021/acsami.9b00334, 2019.

[5] A. M. Steiner et al., "Macroscopic Strain-Induced Transition from Quasi-infinite Gold Nanoparticle Chains to Defined Plasmonic Oligomers," ACS Nano, vol. 11, pp. 8871-8880, 2017. 